\title{
DUNE's Potential to Search for Neutrinoless Double Beta Decay
}

Elise Chavez, University of Wisconsin-Madison - GEM Fellow

Joseph Zennamo and Fernanda Psihas, Fermilab

WISCONSIN

FERMILAB-POSTER-20-104-ND

\section{INTRODUCTION}

Neutrinoless double beta decay is a forbidden process by the standard model due to a violation of lepton number and could be piece of the matter/anti-matter asymmetry puzzle. Its rarity makes it very difficult to search for, but if found could aid in figuring out the mass hierarchy. The idea is to take advantage of DUNE's size and capabilities to attempt to detect the decay by doping the liquid argon with Xenon, a candidate isotope for this decay. Thus, we need to know whether this search is feasible within DUNE's framework.

\section{PURPOSE}

To analyze and model the potential backgrounds in the detector that come from radiologic decays in the detector that are caused by instability, spallation caused by cosmic muons, solar neutrinos, and the environment to aid in determining whether this type of search is feasible.

\section{METHODS}

To achieve the project goals, a simulation file has been analyzed that analyzed the radioactive decays in the LArTPC. The sources have come from the cathode, anode, Polonium, Krypton, Argon 42, and neutron. Much of this project has also been learning how to use DUNE's framework from art to LArSoft. An analyzer module had to be made to filter down the data in the file. This ensures that the results are still applicable to DUNE.
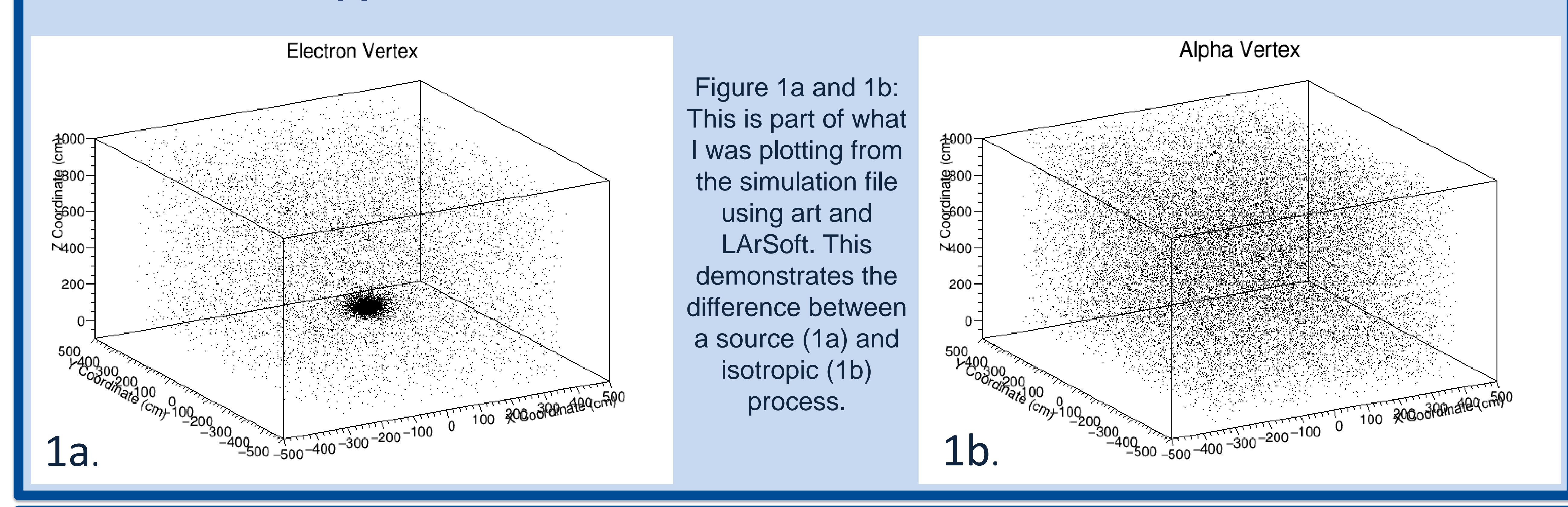

\section{CONCLUSIONS}

Most of what I see from the file is electrons, which is what the signal will consist of. The signal being two energic electrons. There are also many photons and alphas coming from the radiologic decays. Future steps include examining the motherage of these particles to better understand their abundance and ability to disguise the desired signal, along with an analysis of a background file, different from a source file, and a subsequent comparison. This will allow for a better conclusion and better understanding of the potential of this search in the future.

Figure 1: A
diagram of
neutrinoless
double beta decay
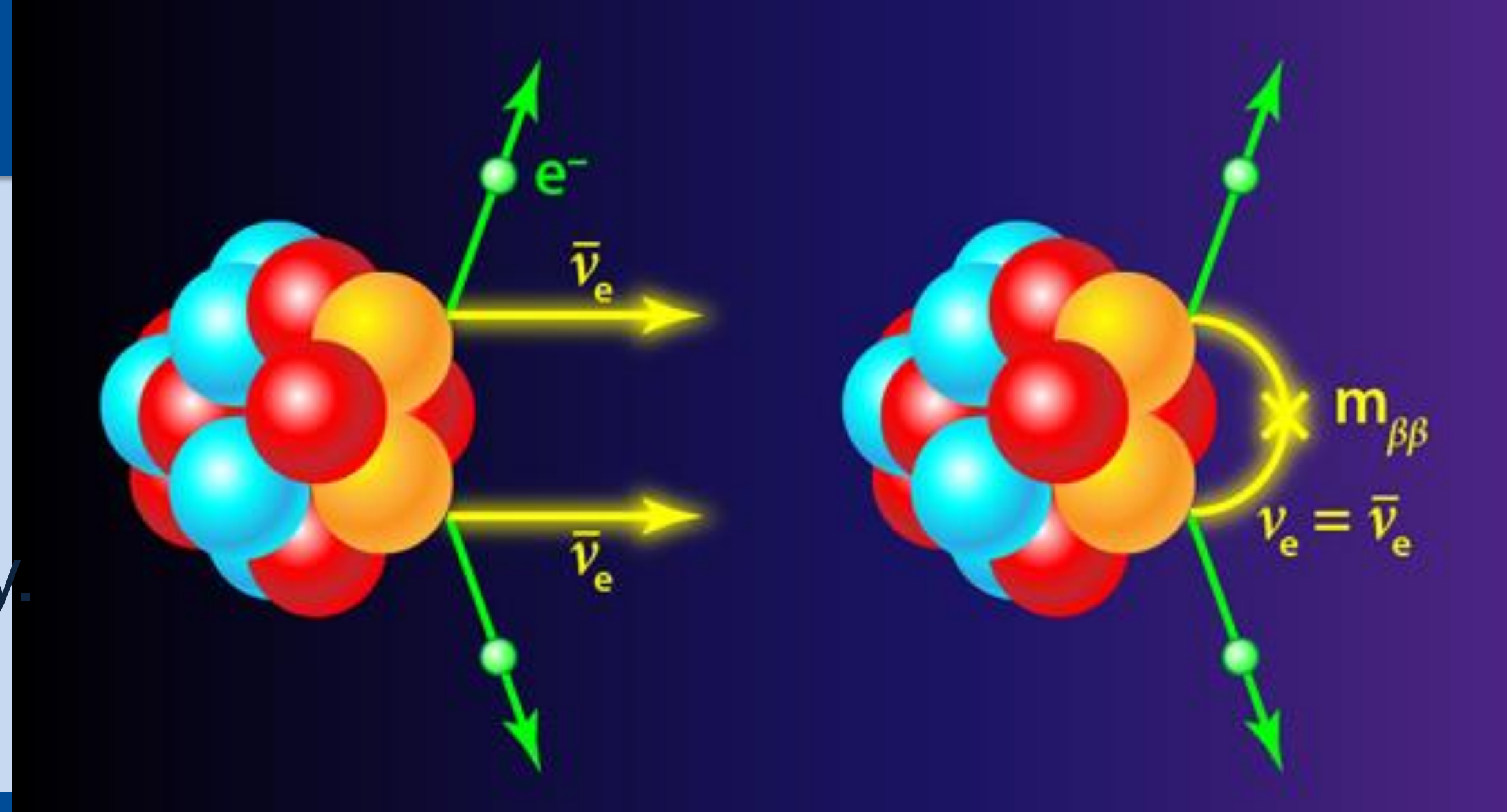

RESULTS

The results given here are the plots described in methods. These are the plots for the backgrounds with significant statistics: positrons, photons, and electrons.
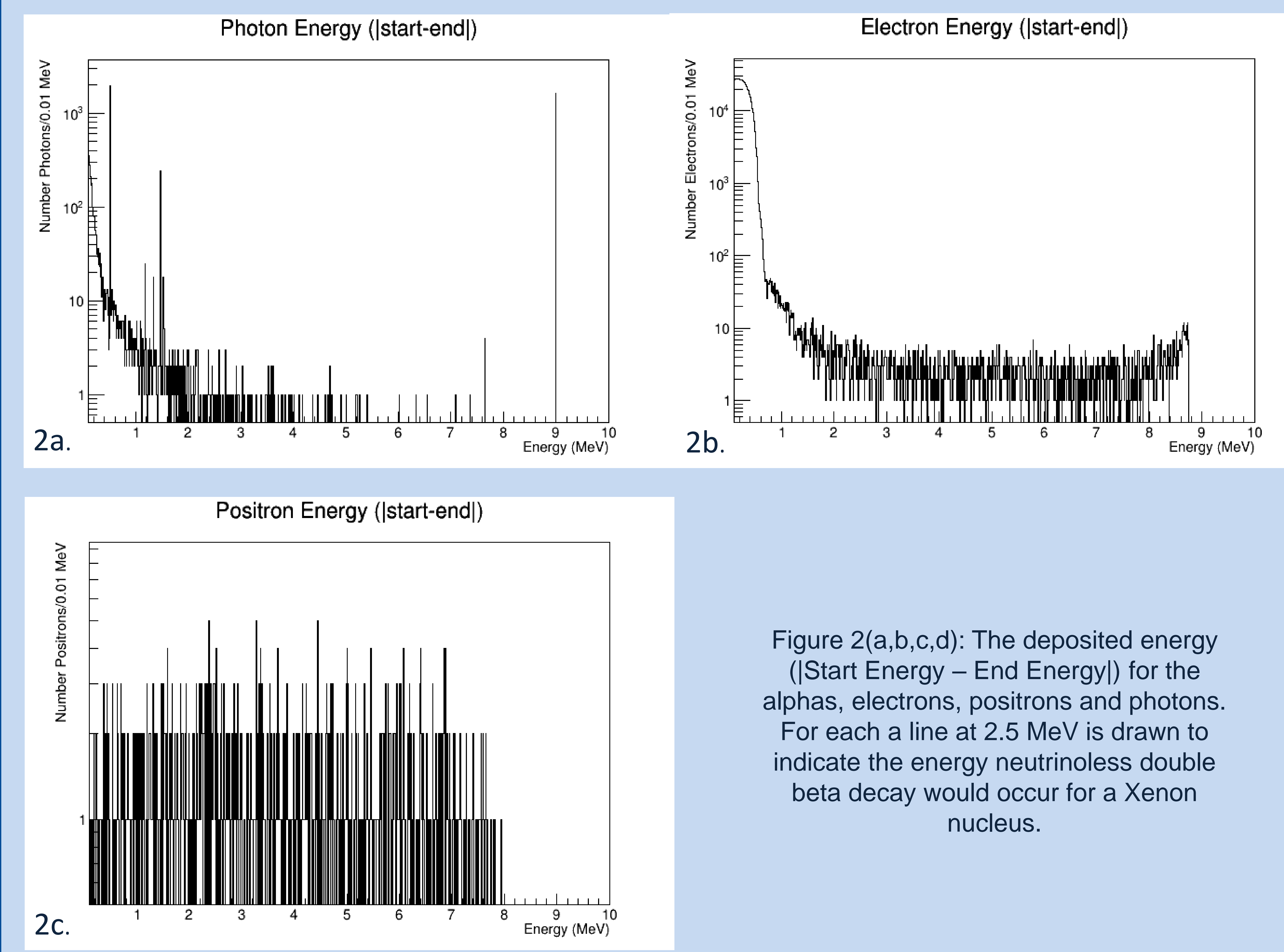

Figure 2(a,b,c,d): The deposited energy (|Start Energy - End Energy|) for the alphas, electrons, positrons and photons. For each a line at $2.5 \mathrm{MeV}$ is drawn to indicate the energy neutrinoless double nucleus. beta decay would occur for a Xenon 